Número DOI: 10.1590/permusi2015b3213

ARTIGO CIENTÍFICO

\title{
Lana Tai - no dia em que nasceu uma aquarela: diário de bordo de uma composição a partir de descritores de áudio
}

\section{“Lana Tai - no dia em que nasceu uma aquarela”: composing via audio descriptors}

\author{
Ivan Eiji Simurra ${ }^{1}$ \\ Jônatas Manzolli ${ }^{2}$
}

\author{
${ }^{1}$ Núcleo Interdisciplinar de Comunicação Sonora (NICS), Departamento de Música (DM/IA) \\ Universidade Estadual Campinas - Campinas, São Paulo, Brasil \\ ieysimurra@nics.unicamp.br \\ ${ }^{2}$ Núcleo Interdisciplinar de Comunicação Sonora (NICS), Departamento de Música (DM/IA) \\ Universidade Estadual Campinas - Campinas, São Paulo, Brasil \\ jonatas@nics.unicamp.br
}

\section{Resumo}

Trata-se do relato de um processo criativo musical no qual utilizou-se estratégias composicionais vinculadas a análises de conteúdo espectral com descritores de áudio. Tal procedimento foi o suporte para a elaboração da obra "Lana Tai - no dia em que nasceu uma aquarela", para orquestra de cordas. A partir de um banco de dados sonoros, realizaram-se diversas experimentações com o objetivo de testar configurações orquestrais elaboradas com técnicas estendidas de execução instrumental. Tais experimentos foram realizados com suporte do ambiente computacional Pure Data (PD) com o qual utilizou-se a biblioteca de descritores de áudio PDescriptors. Os resultados, as reflexões e as questões levantadas durante toda a elaboração, criação e execução de "Lana Tai" suscitam um novo aporte no desenvolvimento tanto da pesquisa em processos criativos quanto na criação de novas obras inéditas.

Palavras-chave: Composição Musical; Orquestração Assistida por Computador; Técnicas Estendidas;

Descritores de Áudio; Pure Data.

\begin{abstract}
This article is a review of a music creative processes in which it was applied compositional strategies combined to sound spectral analysis based on audio descriptors. This procedure supported the creation of the work "Lana
\end{abstract}


Tai - no dia em que nasceu uma aquarela" for string orchestra. For that, some instrumental mixtures and orchestration were made using a Sound DataBase. In this particular case, a virtual environment in Pure Data (PD) software, using the library for analysis fucntions PDescriptors, analyzed some potential orchestral settings. All the results, issues and compositional reflections were used to create "Lana Tai - no dia em que nasceu uma aquarela". This approach rises up a new contribution in the development of research in both creative processes and the creation of new unpublished works.

Keywords: music composition; computer-aided orchestration; extended techniques; audio descriptors; Pure Data.

Data de recebimento: 07/03/2015

Data de aprovação final: 22/09/2015

\section{1 - Introdução}

$\mathrm{Na}$ atualidade, o compositor dispõe de vários recursos sonoros e tecnológicos para criar a sua obra, seja para instrumento solo ou para orquestra sinfônica. Dentro desse escopo, este artigo apresenta uma composição para orquestra de cordas criada com suporte computacional. Nossa pesquisa foca a composição musical relacionada às transformações do timbre musical que alteram sensivelmente as características espectrais de cada nota ouvida. Esses procedimentos de transformação sonora podem ser comparados à pintura de uma aquarela tal como foi arquitetada na obra "Lana Tai - no dia em que nasceu uma aquarela". Seria como se uma paleta de cores fosse misturada por técnicas instrumentais estendidas e produzisse novos matizes das quais alteram a sonoridade esperada do timbre da orquestra de cordas. O concerto do qual "Lana Tai" participou foi realizado pela Orquestra Sinfônica da Unicamp, em outubro de 2012, sob a regência do maestro Akira Miyashiro².

No âmbito deste trabalho, "suportes tecnológicos" devem ser interpretados como ferramentas auxiliares ao processo composicional. A área de desenvolvimento tecnológico que interage com nosso estudo denomina-se Music Information Retrieval ou MIR, como

\footnotetext{
1 A partir deste ponto utilizaremos o título "Lana Tal" para nos referirmos à obra aqui reportada.
}

2 Para mais informações: http://tinyurl.com/lana-concerto, acesso: 22/05/2013. 
descrito por BYRD e FINGERHUT (2002). Nela, utilizam-se recursos de processamento digital de sinais para extrair características sonoras do espectro do som. Na literatura especializada na área, tais medidas de características são denominadas "descritores de áudio". Como descrevem TZANETAKIS e COOK (2002), os procedimentos relacionados à classificação automática de sinais musicais localizam, indexam e catalogam objetos sonoros/musicais de acordo com diversos critérios paramétricos. Em particular, Tzanetakis emprega três critérios: 1) textura timbrística, 2) discriminação de altura e 3) conteúdo rítmico. Na pesquisa aqui reportada são utilizados dois descritores de áudio, o Croma e o Centroide, que dialogam com os primeiros critérios discutidos por Tzanetakis. Outra aplicação importante, com a qual o nosso trabalho dialoga, é a pesquisa de MALT e JOURDAN (2009), que utiliza descritores de áudio para analisar as técnicas estendidas presentes no repertório da música do século XX. De forma similar, analisamos neste artigo diversas técnicas estendidas dos instrumentos de cordas. Esse procedimento será apresentado no final deste texto no qual discutimos os "Marcos Sonoros", que foram construídos com excertos contendo vários modos de ataque em diferentes registros.

Do ponto de vista tecnológico, os procedimentos desenvolvidos por pesquisadores, tais como PAMPALK (2006) e BOGDANOV; WACK e GOMEZ (2011), deram origem a novos ambientes computacionais para realizar análises de áudio nas quais quantificam-se diversas categorias descritivas do sinal sonoro, tais como conteúdo espectral, tonal e rítmicos. O trabalho dos pesquisadores LAMBROU; KUDUMAKIS; SPELLER; SANDLER e LINNEY (1998), concentrou-se na extração de alguns parâmetros a partir de domínios temporais e da transformada Wavelet para descrever o comportamento rítmico em diferentes gêneros musicais, tais como jazz e rock. De maneira análoga, no trabalho de DESHPANDE; SINGH e NAM (2001), a classificação foi realizada por intermédio de uma análise gráfica, via espectrogramas, para o reconhecimento e classificação dos mesmos gêneros musicais, rock e jazz. BARBEDO e LOPES (2007) elaboraram uma importante classificação de gêneros musicais, tais como rock, erudito e dance music, a partir de uma taxonomia hierárquica relacionada com valores paramétricos extraídos dos sinais musicais analisados. THOSHKAHNA; NSABIMANA e RAMAKRISHNAN (2011), automatizaram a remoção de ruído sonoro; o trabalho de DIELEMAN e SCHRAUWEN (2013) realiza a complexa tarefa de separar diferentes fontes de áudio num mesmo sinal digitalizado; CARTWRIGHT 
e PARDO (2013) utilizam os recursos da tecnologia MIR para controlar procedimentos de mixagem e equalização sonora no estúdio digital. Ademais, as pesquisas acerca de descritores de áudio são emergentes e há iniciativas singulares como a proposta do projeto CUIDADO - Content-based Unified Interfaces and Descriptors for Audio/music Databases avaliable online (PEETERS, 2004), para padronizar as características do comportamento acústico do sinal sonoro no reconhecimento da fala e da música. Outro projeto pioneiro, o qual concentra-se na recuperação da informação musical, denomina-se Music Information Retrieval Evaluation eXchange ou MIREX, descrito por DOWNIE; EHMANN; BAY e JONES (2010), que é um conjunto de técnicas para avaliar os desenvolvimentos científicos, os sistemas e os algoritmos de recuperação da informação musical.

Em síntese, o processo criativo aqui reportado partiu de dois universos: a) as técnicas de execução instrumental estendidas e b) as ferramentas computacionais para analisar e descrever estatisticamente o conteúdo espectral do material gerado por essas técnicas. Assim, desenvolvemos um método para auxiliar o compositor a relacionar: a) as descrições de alto nível ou simbólicas, aqui denominadas de "sonoridade" com b) os modos específicos de execução instrumental estendida. No caso de "Lana Tai", foram exploradas duas ideias contrastantes: a) Sonoridades Opacas e b) Sonoridades Brilhantes. Esperamos estabelecer, por intermédio de tal metodologia, uma mescla mais refinada do material composicional enquanto fenômeno timbrístico. O termo "mescla", insere-se no âmbito de misturas instrumentais, ou seja, a interação da orquestração com as diversas técnicas de execução. Há diversas relações complexas entre o "material sonoro", os "materiais simbólico-musicais" e as construções timbrísticas e orquestrais. No estudo aqui reportado, orquestração é um sistema de distribuição de índices, claramente descritos em notação musical a partir de um conjunto simbólico, os quais são relacionados com instrumentos, notas musicais, dinâmicas, técnicas e modos de execução instrumental. A partir desse modo de operar sobre tais elementos simbólicos, dependendo substancialmente dos modos de execução instrumental, a partitura orquestral é projetada como um todo dentro de um espaço de possibilidades sonoras à qual, no âmbito dessa resultante orquestral, pode ser pouco previsível, justamente por abarcar características outras, extrapolando a esfera das propriedades simbólicas da notação musical. O objetivo deste trabalho centrou-se em ajustar os índices com as possibilidades de execução instrumental derivadas de técnicas estendidas. De maneira 
intuitiva, pode-se dizer que a orquestração com a qual trabalhamos nesse artigo compreende uma mistura dos mais variados timbres e técnicas de execução instrumental.

As próximas seções apresentam um breve relato do diálogo entre orquestração musical, criação no século XX e suporte tecnológico (Seção 2). A seguir, recapitulamos a literatura sobre os descritores de áudio com o objetivo de introduzir os dois descritores utilizados no nosso estudo (Seção 3). Na Seção 4, descrevemos o planejamento composicional de "Lana Tai " no qual definimos os conceitos "Pontos de Referência" (PR) e os "Marcos Sonoros" (MS). A Seção 5 discute os MS a partir do Croma e do Centroide. Na Seção 6, fazemos projeções futuras e concluímos o artigo.

\section{2 - Orquestração e Suporte Tecnológico}

Os procedimentos reportados nessa seção sintetizam o estudo contemporâneo sobre orquestração com suporte computacional. Segundo CARPENTIER (2008, p.37), as questões relacionadas às estratégias de orquestração, dentro da História da Música, sempre encontraram-se ancoradas na subjetividade, empirismo e nas "práticas comuns" desenvolvidas pelos próprios compositores. Apesar de historicamente relevantes, mesmo os tratados de instrumentação e orquestração consagrados, ainda não apresentam uma discussão apoiada em critérios objetivos. Dentre eles, destacam-se os trabalhos de BERLIOZ (1844), RIMSKY-KORSAKOV (1912) e PISTON (1955).

Por outro lado, já em meados da década de 1980, o compositor e regente Pierre Boulez projetava a importância do timbre na música instrumental do século XX e a iminente colaboração dos avanços tecnológicos aplicados à orquestração musical. BOULEZ (1987) ratifica tal abordagem ao considerar o timbre de um ponto de vista também objetivo e cientifico. Como observou Boulez (1987, p.162), do ponto de vista artístico e subjetivo, o timbre é uma propriedade da linguagem musical, concomitante a estética e a forma musical. Contudo, tal abordagem impossibilita o relacionamento da "intuição qualitativa" do timbre com a "avaliação quantitativa das categorias de medidas e análises objetivas do 
comportamento do timbre musical". No nosso ponto de vista, estudar o timbre musical do ponto de vista científico, vincula-se indiretamente com uma abordagem poética e musical. Pois, as pesquisas acerca das características objetivas das microestruturas espectrais internas do timbre musical aprofundam o desenvolvimento de uma escuta mais refinada e consciente. As relações e as características do comportamento do espectro sonoro devem ter um lugar significativo e consciente no projeto particular de cada compositor, independente de sua estética ou poética musical. Para o compositor JONATHAN HARVEY (1980, p.699), a organização espectral da música deve ser muito bem elaborada, pois as relações das alturas musicais com suas componentes espectrais são bastante difusas e complexas. O questionamento de Harvey concentra-se, principalmente, quando utilizam-se instrumentos de percussão no repertório contemporâneo. Ele cita como exemplo a análise do comportamento espectral de um prato suspenso que apresenta componentes espectrais complexas, intrínsecas à sua sonoridade.

Há estudos que vinculam a orquestração com a representação do espectro sonoro. Para PIRES (2011, p.42), o timbre orquestral é a resultante da evolução temporal das componentes espectrais geradas pela interação de múltiplos instrumentos e técnicas. Uma visão pioneira de tal ponto de vista foi desenvolvida por MANZOLLI (1988), utilizando modelagem matemática e métodos de otimização aplicados ao estudo da orquestração. Nesse estudo, dada uma "função-critério", o autor desenvolveu um procedimento computacional que disponibilizava ao compositor os parâmetros de análise necessários à obtenção do efeito timbrístico desejado. Essa relação é descrita no trabalho pela distância euclidiana ${ }^{3}$ de duas funções: a "função-timbre objetivo" e a "função-timbre orquestral". A primeira quantifica objetivamente o equilíbrio desejado e a segunda descreve esse timbre a partir do espectro sonoro. Essa, estrutura-se pela superposição de "funções-timbre de cada instrumento" que são construídas com as Curvas de Envelope Espectral e de Loudness ${ }^{4}$ de cada instrumento musical analisado. Por Envelope Espectral, MANZOLLI (1998, p.42) entende uma

\footnotetext{
3 Calcula-se a distância euclidiana de $n$-pontos de vetores diferentes a partir da seguinte equação: $D=\sqrt{\left(P_{x}-Q_{x}\right)^{2}+\left(P_{y}-Q_{y}\right)^{2}+\ldots+\left(P_{n}-Q_{n}\right)^{2}}$ 4 Loudness pode ser definido como a medida psicoacústica relativo à percepção de intensidades sonoras.
} 
persistência nos padrões de energia e de quantidade de componentes espectrais de um sinal sonoro analisado.

Posteriormente, a partir dos trabalhos de PSENICKA (2003), HUMMEL (2005) e ROSE e HETRICK (2009), há uma evolução da orquestração assistida por computador, na qual destacam-se trabalhos que, de forma similar a Manzolli, utilizam-se de modelos matemáticos associados ao envelope espectral dos instrumentos da orquestra com a otimização da soma desses envelopes para descrever um timbre-alvo. Há ainda, ambientes computacionais os quais concentram-se na busca das melhores soluções orquestrais entre um banco de amostras sonoras e um timbre-alvo, o qual pode ser estabelecido por intermédio de uma gravação sonora ou um som sintetizado digitalmente. Nos trabalhos de CARPENTIER (2008), ESLING; CARPENTIER e AGON (2010) e; ESLING e AGON (2013), a busca por um timbre alvo é realizada pelas características espectrais e parâmetros musicais dos sinais analisados, tais como altura musical, frequência sonora da fundamental, amplitude dos componentes espectrais, dentre outros. Da mesma forma que em CARPENTIER (2008), o nosso trabalho utiliza-se de um banco de amostras sonoras digitais para construir os Marcos Sonoros (MS). A nossa proposta é utilizar o próprio som como elemento potente do processo criativo. Dessa forma, descrevemos a nossa abordagem como sendo a aplicação de descritores de áudio como suporte à Orquestração Assistida por Computador. Na próxima Seção, fazemos uma breve revisão da literatura sobre os dois descritores de áudio utilizados na nossa pesquisa.

\section{3 - Descritores de Áudio}

Uma das alternativas de expansão das ferramentas de análise musical são algoritmos computacionais que descrevem características do comportamento do espectro do som digitalizado. Nesta seção, de modo complementar às referencias já citadas na introdução do artigo, apresentamos outras com as quais dialogam mais diretamente com o nosso estudo. Como já apresentado, a área de conhecimento científico sobre o assunto denomina-se "Music Information Retrieval" ou simplesmente, MIR. Os estudos sobre MIR utilizam funções 
matemáticas, apoiadas em medidas estatísticas e modelos psicoacústicos para desenvolver os chamados descritores de áudio. Segundo PEETERS (2004, p.01), os procedimentos para descrever as características de um sinal sonoro foram propostos pela comunidade científica para o reconhecimento de padrões da fala e para a classificação de instrumentos musicais. Esses procedimentos também são ferramentas de relevância significativa no contexto da composição e orquestração musical assistidas por computador, como já comentados na Seção anterior. Os métodos para analisar o conteúdo espectral, a partir de sinais de áudio digitalizados, são realizados via Short-Time Fourier Transform ou STFT, que é definida a seguir por SHEH e ELLIS (2003, p.02):

$$
X_{\text {STFT }}=[k, n]=\sum_{m=0}^{N-1} x[n-m] * w[m] * e^{-j 2 \pi k m / N}
$$

onde, $k$ indexa o eixo de frequência (bin) dentro do intervalo $0<k<\mathrm{N}-1$ do $n^{\text {ésimo }}$ quadro; $w[m]$ é uma janela de $N$ amostras.

No âmbito da utilização de ambiente computacional de assistência à análise e à composição musical, McADAMS e BATTIER (2005) desenvolvem o trabalho de análise, via descritores de áudio da obra "The Angel of Death", do compositor Roger Reynolds. Como já mencionamos, o trabalho de MALT e JOURDAN (2009, p.6-8) investiga a identificação, via descritores de áudio, de diversas técnicas instrumentais estendidas, tais como os ruídos de chaves (key clicks), no saxofone alto e as articulações particulares do fagote. Dentro deste escopo, vamos nos concentrar na definição dos dois descritores utilizados no nosso estudo: Croma Espectral e Centroide Espectral.

\section{1 - Croma Espectral}

O Croma Espectral (que é denominado apenas de Croma no restante do artigo) é caracterizado pela distribuição estatística acumulada da magnitude do espectro de Fourier, por bandas fixas de frequência. Essas, referem-se às classes de alturas da escala cromática. Originalmente, o cálculo do Croma foi descrito no trabalho de FUJISHIMA (1999), que o denominou como perfil de classes altura, Pitch Class Profile ou PCP. O descritor foi muito utilizado na extração de informações relacionadas com estruturas harmônicas e tonalidade. O seu modelo perceptivo baseia-se na pesquisa de SHEPARD (1982), no qual definiu-se 
duas características importantes para o reconhecimento de hierarquias de alturas musicais: a) a configuração rotacional denominada de Croma ou "Pitch Class", definida como a equivalência da percepção das alturas musicais independente do seu registro ou oitava (vide Figura 1a) e; b) A posição vertical do índice do Croma, o qual Shepard chama de altura ou "height", à qual é apresentada numa espiral, tal como ilustrado na Figura $1 b$.
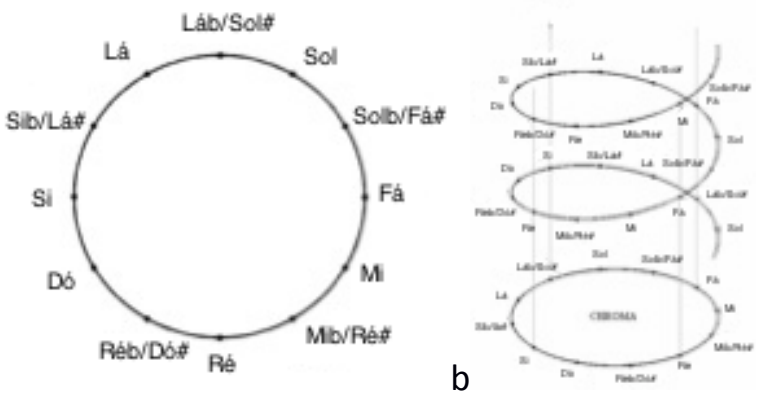

Figura 1: Representação do comportamento rotacional (a) e espiral das alturas em oitavas diferentes (b).

Gomez (2006) atualizou o algoritmo PCP, implementando o "Harmonic Pitch Class Profile", HPCP. Nesta atualização, implementou-se a ponderação $\log _{2}$ para caracterizar a propriedade logarítmica da percepção de alturas musicais com o objetivo de destacar a contribuição das componentes harmônicas de cada altura musical. Ou seja, para a escala cromática de 12 notas, os componentes harmônicos são calculados pelo valor inteiro dado pelo calculo do módulo 12 em relação à frequência fundamental. Com esse procedimento, focou-se na resolução da distribuição da energia em classes de alturas. Utilizando-se o HPCP é possível configurar outras bandas de frequência que extrapolam a escala cromática tais como quartos de tom e sextos de tom. Com isso, os bins $k$ de uma $S T F T$, vide Eq. 1, são relacionadas com os $P[k]$ do Croma da seguinte forma:

$$
P[k]=\left[12 \log _{2} \frac{k}{N} \frac{f_{s r}}{f_{\text {ref }}}\right] \bmod 12(2)
$$

onde $f_{\text {sr }}=$ frequência de amostragem, $f_{\text {ref }}=$ frequência de referência à qual, pela convenção desse algoritmo, estabelece o valor de $440 \mathrm{~Hz}$ para a classe da nota Lá4. O valor 12 na Eq. 2 está associado às notas da escala cromática. Esse valor deverá ser modificado no caso que outras subdivisões temperadas, como por exemplo: o valor 24 para quarto de tom e 36 para sexto de tom. A partir da Eq. 2, são calculados os valores da distribuição do Croma que são 
dados pela soma das magnitudes de todos os bins de frequência $\mathrm{P}[\mathrm{k}]$ que correspondem a cada uma das classes de alturas:

$$
\operatorname{Chroma}[j]=\sum_{k: P(k)=j}^{12}|X[k]|^{2}(3)
$$

onde: $j$ é o índice relacionado à resolução das classes de alturas. Para semitom $\mathrm{j}=0,1, \ldots$, 11 , quartos de tom $\mathrm{j}=0, \ldots, 23$ e para sexto de tom $\mathrm{j}=0, \ldots, 35$. Vide que o somatório varia dentro dos índices determinados pela Eq. 2. A Figura 2, a seguir, apresenta o esquema geral do algoritmo de Croma, segundo o diagrama de FUJISHIMA (1999).

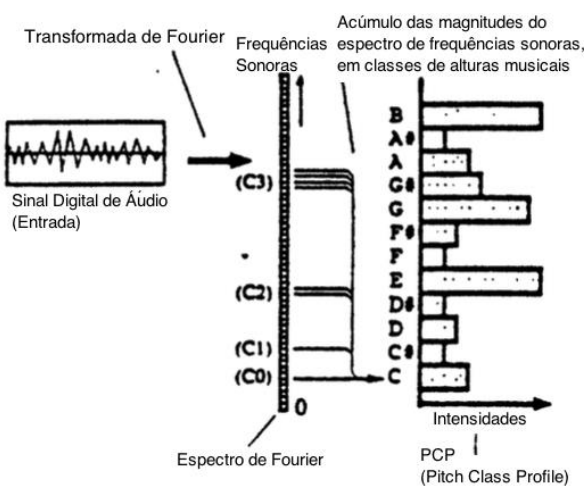

Figura 2: Esquema geral do algoritmo de Croma.

\section{2 - Centroide Espectral}

Segundo THEODORIDIS e KOUTRUMBAS (2006, p.460-461), um dos parâmetros mais utilizados para a extração de características do sinal sonoro é o Centroide Espectral (que é denominado apenas de Centroide no restante do artigo). Tal como apontado por PEREIRA (2009, p.16), por intermédio do Centroide é possível medir a percepção de "brilho" do sinal sonoro analisado. Analogamente, tal descritor pode ser definido como o centro de massa ou baricentro do espectro analisado. Dessa forma, quanto mais alto o valor do Centroide, dado em Hz, maior será a concentração de energia nas componentes espectrais de altas frequências o que pode levar, em muitos casos, à percepção de um som mais “brilhante". Cabe ressaltar que o registro e a altura musical não relacionam-se diretamente com o Centroide. Por exemplo, uma nota tocada no grave, pode apresentar um valor de Centróide em frequências altas. Isto ocorrerá se as magnitudes de seus componentes espectrais de alta frequência tiverem mais energia que os de baixa. O uso de técnicas estendidas pode produzir transientes que elevam o valor da energia nas componentes de alta frequência. Em "Lana Tai", 
exploramos justamente esse tipo de contraste do qual é resultante da superposição de diferentes modos de ataque nos instrumentos de corda. O Centroide é definido pela seguinte equação:

$$
\mathrm{SC}_{(i)}=\frac{\sum_{k=1}^{K-1} f(k) \bullet\left|X_{i}(k)\right|^{2}}{\sum_{k=1}^{K-1}\left|X_{i}(k)\right|^{2}}
$$

onde, $\left|\mathrm{X}_{\mathrm{i}}(\mathrm{k})\right|$ representa a magnitude da $\mathrm{k}^{\text {ésima }}$ componente espectral de uma Transformada Discreta de Fourier de quadro $i$ e K é a metade do número de componentes adotados para a Transformada Discreta de Fourier, ou seja, é a metade do número de amostras da janela de análise; $f(k)$ é a frequência centro do componente $k$, definida como:

$$
f(k)=\frac{\text { taxadeamostragem }}{2 K} k(5)
$$

No nosso trabalho, a extração do Centróide foi realizada em PureData com a biblioteca PDescriptors ${ }^{5}$, desenvolvida por MONTEIRO (2011) no Núcleo Interdisciplinar de Comunicação Sonora - NICS/UNICAMP. Em suma, as técnicas de recuperação da informação musical via descritores relacionam o comportamento do espectro sonoro com propriedades sonoras e foram aplicadas no planejamento composicional de "Lana Tai" para: a) descrever as características do comportamento do espectro sonoro e relacioná-las com b) os modos específicos de execução instrumental estendida utilizadas na orquestração. Inicialmente, o processo composicional concentrou-se em elaborar a trajetória da sonoridade da obra, ou seja, descrever os "Pontos de Referência" que serão apresentados na Seção 4.2.

\section{4 - Processo Criativo de "Lana Tai"}

O processo composicional de "Lana Tai" foi dividido em quatro etapas e apoiou-se em dois conceitos que definem o planejamento formal inicial e a arquitetura da obra. O primeiro

5 Para mais informações: Apple Logic Pro 9. Disponível em http://www.apple.com/logicpro/resources/. Data de acesso: 07/09/2015. 
conceito, denominado "Pontos de Referência" é definido como demarcações que o compositor realiza a priori no seu planejamento composicional, utilizando os descritores de áudio para construir um espaço de sonoridades. Ou seja, são as demarcações que apontam para características timbrísticas potenciais que são associadas aos descritores de áudio. $\mathrm{O}$ segundo conceito, denominado de "Marcos Sonoros ${ }^{6} "$, é definido como as diversas simulações que são feitas com o computador para testar misturas instrumentais que estão relacionadas aos "Pontos de Referência”. Os MS foram gerados com registros sonoros digitais de diversos modos de ataque, articulações e técnicas estendidas instrumentais. As amostras sonoras utilizadas em "Lana Tai" foram as compiladas por BAILLET; BORGHESI; HOFFMANN e LEVY (1999).

\section{1 - O Processo}

O processo de composição da obra relaciona-se com quatro etapas distintas: na Etapa 1, definimos as características timbrísticas a serem exploradas por intermédio dos dois descritores de áudio: Croma e Centroide (vide Seção 3). Para aplicar o Croma, utilizou-se como referência a classe de altura Ré\#. O Centroide, foi utilizado para apontar configurações brilhantes e opacas. O projeto inicial desenvolveu-se a partir da ideia de criar-se variações de sonoridades brilhantes ou opacas iniciadas no Ré\# e espalhadas por outras alturas. Na Etapa 2, utilizando-se os dois conceitos de sonoridade da Etapa 1, elaboramos os "Pontos de Referência". Essas demarcações definiram a trajetória da obra por intermédio da manipulação do Centroide. Na Etapa 3, simulamos os "Marcos Sonoros" com o computador, utilizando-se amostras sonoras digitais de diversos modos de ataque, articulação e técnicas estendidas dos diversos instrumentos da família das cordas. Por fim, na Etapa 4 são efetuadas as escolhas das configurações orquestrais ponderadas pela interpretação do compositor e os valores da distribuição do Croma. Junto com a estratégia utilizada para a escolha do efetivo instrumental, levou-se em consideração as semelhanças e as particularidades de cada instrumento do conjunto (violinos, violas, violoncelos e contrabaixos), utilizados como instrumentos solistas ou como pertencentes a um subconjunto instrumental particular. A escolha de tal efetivo instrumental deu-se por três fatores: 1) "Foco Timbrístico": optou-se

6 Cabe ressaltar que o termo "Marcos Sonoros" desenvolvido na nossa pesquisa, não se relaciona conceitualmente com o termo Sound Marks apresentado por M. Schaffer (1977) ao tratar de paisagens sonoras. 
por uma redução na complexidade sonora de uma orquestra sinfônica completa. Contudo, a utilização de todos os membros da família das cordas propiciou vários parâmetros de controle da paleta espectral desses instrumentos, ou seja, as possibilidades de misturas desde os contrabaixos até os violinos; 2) "Maior dimensão das texturas sonoras resultantes": ao utilizar um número maior de instrumentos (maior que o de um quarteto de cordas, por exemplo), a obra explorou resultados timbrísticos mais complexos, com maior controle de nuances sonoras e; 3) "Instrumentação e técnicas estendidas instrumentais particulares do naipe das cordas": explorou-se as particularidades dos modos de execução dos instrumentos de cordas e, mais ainda, as peculiaridades de cada instrumento participante desse efetivo.

\section{2 - Elaboração dos Pontos de Referência}

A elaboração dos Pontos de Referência apoiou-se em três etapas distintas, na qual a Etapa1 inicia a trajetória composicional executando uma nota musical em uníssono, a nota Ré\#4. $\mathrm{Na}$ Etapa 2, desenvolveu-se gradativamente as configurações orquestrais com baixo valor do Centroide para caracterizar sonoridades opacas. Na Etapa 3, finalizamos a trajetória com configurações orquestrais com alto valor de Centroide para caracterizar sonoridades brilhantes. No planejamento inicial, determinou-se que a obra tivesse 15 Pontos de Referência (PR), os quais se relacionam com as três etapas apresentadas acima. O PR 06 apresentou o menor valor para o Centróide e o PR 15 o seu maior valor. As transições entre os PR's agregaram gradualmente diferentes técnicas estendidas, ampliando gradativamente o número de notas e a tessitura de cada naipe. A Figura 3 representa a trajetória das transformações timbrísticas que descrevem a sonoridade global de "Lana Tai":

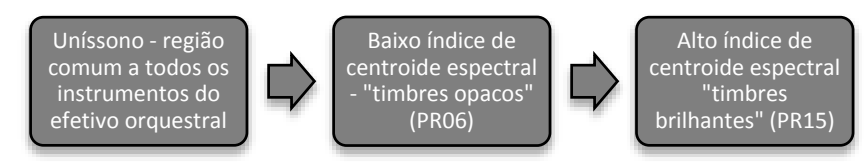

Figura 3: Diagrama geral do percurso timbrístico em Lana Tai.

Como já mencionamos acima, os Pontos de Referência, conduziram a construção dos Marcos Sonoros. Tais configurações foram geradas com registros sonoros digitais. A próxima Seção descreve os procedimentos utilizados para elaborá-los. 


\section{3 - Os Marcos Sonoros}

Os Marcos Sonoros (MS) foram gerados com a ajuda de suporte computacional e com amostras de um banco sonoro digital. Esse banco possui amostras curtas, com duração média entre cinco e sete segundos armazenadas no formato .aiff. Essas amostras são gravações de execuções instrumentais com diversas técnicas estendidas com diferentes modos de ataque, alturas e intensidades. Os MS foram gerados misturando-se as amostras com o software Logic Pro $^{7}$ e depois de criá-los, foi possível calcular os valores do Croma e Centroide Espectral para cada um deles. A Figura 4, abaixo, apresenta a interface do programa e a janela com os MS.

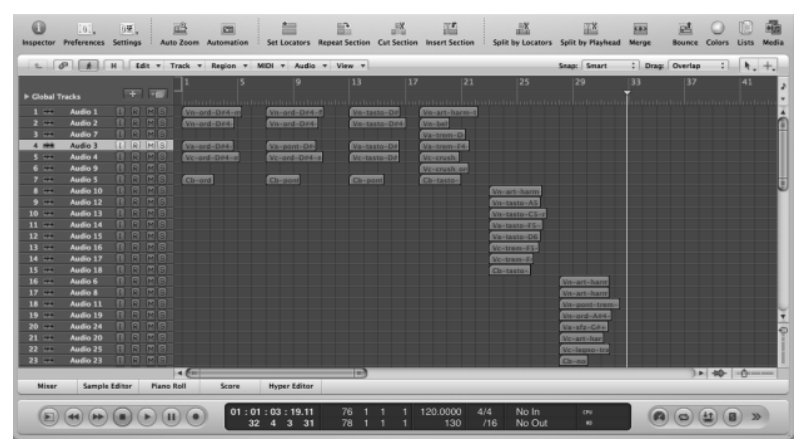

Figura 4: Figura apresentando 6 MS gerados com amostras áudio digital.

\section{4 - Procedimentos de Transcrição}

O processo composicional concentrou-se em estabelecer relações de proximidade e distanciamento entre os Pontos de Referência e os Marcos Sonoros. Os PR estabeleceram o ideal sonoro planejado pelo compositor no momento da concepção da obra. Já os MS reproduziram a construção possível com o material timbrístico construído com as simulações computacionais. Todas as etapas da geração dos MS foram realizadas empiricamente, em sua essência e todos os 15 MS foram analisados com a biblioteca PDescriptors (MONTEIRO, 2011). O critério final para a escolha das amostras sonoras foi ponderado pela experiência do próprio compositor.

7 Para mais informações: Apple Logic Pro 9. Disponível em http://www.apple.com/logicpro/resources/. Data de acesso: 07/09/2015. 
Cada MS apresentou uma distribuição diferente do Croma. Cada uma delas está relacionada com as mudanças de registro, alturas de referência e os modos de execução e de instrumentos. Esse procedimento foi utilizado para ampliar, via técnicas instrumentais estendidas, as possibilidades timbrísticas dentro de um âmbito espectral previamente estabelecido. Produziu-se resultantes espectrais relacionadas com características inerentes ao comportamento acústico de cada instrumento. Para a transcrição final da obra, foram escolhidos os MS com os maiores valores no histograma do Croma e as classes de altura, a eles associadas, foram utilizadas na transcrição da partitura final (vide Figura 13). A Figura 5 apresenta todos os Marcos Sonoros que foram selecionados para transcrição na obra. A tabela descreve suas respectivas orquestrações, instrumentações e modos de execução. Em suma, a tabela apresentada na Figura 5 foi a referência utilizada para o processo de transcrição dos parâmetros de execução, técnicas estendidas e alturas para a partitura da obra.

\begin{tabular}{|c|c|}
\hline 1 & strings_mf_Ré\#-vn_sp-vn_vasp-vc_cb_sp \\
\hline 2 & vn_ast_Ré\#5-vn_ast_Ré\#4-va_ast_Ré\#3-vc_ast_Ré\#4-cb_asp_Ré\#4 \\
\hline 3 & vn_arthm_tr_Ré7-vn_bb_Si3-va_trem_Ré\#5f-vc_cr_Sol\#3_Ré\#3-cb_ast_Si1 \\
\hline 4 & $\begin{array}{l}\text { vn_harm_ast_Ré\#7-vn_ast_Lá5_Dó5-va_ast_Fá5_Ré6-vc_trem_Fá5_Fá\#4_ast- } \\
\text { cb_ast_Lá\#2 }\end{array}$ \\
\hline 5 & $\begin{array}{l}\text { vn_artharm_Fá7-vn_artharm_Fá7-vn_asp_trem_Dó6-vn_ord_Lá\#4-va_szf_Sol\#+3- } \\
\text { vc_artharm_Dó7-vc_tratto_Lá\#4-cb_nonvib_Fá\#3 }\end{array}$ \\
\hline 6 & vc_sord_nonvib_Lá\#2-vc_sord_nonvib_Fá2-cb_sord_nonvib_Dó+2-cb_sord_nonvib_Fá\#1 \\
\hline 7 & $\begin{array}{l}\text { vn_artharm_trem_Lá\#7-vn_sord_ast_Fá+5-vn_sord_ast_Ré4-va_sord_ord_Lá\#3- } \\
\text { vc_ast_Fá2-cb_ast_Si2 }\end{array}$ \\
\hline 8 & vn_ast_Si5-vn_ast_Si+5-va_ast_Fá+3-vc_ast_Si+2-cb_pizz_Iv_Fá+2 \\
\hline 9 & vn_ast_Sol\#5-vn_ast_Sol5-va_ast_Sol4-vc_nonvib_Si3-cb_ast_Si2 \\
\hline 10 & vn_asp_Ré6-vn_ast_Lá\#5-vn_ast_Sol4-va_ast_Fá\#3-va_asp_Ré\#3-vc_asp_Si2-cb_ord_Si2 \\
\hline 11 & $\begin{array}{l}\text { vn_artharm_Ré\#7-vn_artharm_Lá6-vn_ast_Sol\#5-vn_ord_Ré\#5-va_asp_Dó5- } \\
\text { va_asp_Ré\#4-vc_ast_Fá\#4-vc_ast_Sol4-cb_ord_Dó\#4 }\end{array}$ \\
\hline 12 & $\begin{array}{l}\text { vn_artharm_Ré\#7-vn_artharm_Lá\#6-vn_artharm_Sol6-vn_ord_Ré\#5-va_ast_Fá5- } \\
\text { va_aspd_Ré\#4-vc_asp_Dó5-vc_ast_Mi5-cb_ord_Dó4 }\end{array}$ \\
\hline 13 & $\begin{array}{c}\text { vn_artharm_Sol7-vn_artharm_Ré7-vn_artharm_Fá6-vn_ast_Ré\#6-va_artharm_Ré\#7- } \\
\text { va_artharm_Fá6-vc_artharm_Mi6 }\end{array}$ \\
\hline
\end{tabular}




\begin{tabular}{|c|c|}
\hline 14 & vn_artharm_Ré\#8-vn_artharm_Lá\#7-vn_ord_Fá7 \\
\hline 15 & vn_artharm_Lá\#7-vn_artharm_Ré\#7-vn_artharm_Fá7 \\
\hline
\end{tabular}

Figura 5: As abreviações indicam o instrumento $(\mathrm{vn}=$ violino; $\mathrm{va}=$ viola; $\mathrm{vc}=$ violoncelo e $\mathrm{cb}=$ contrabaixo) e os modos de execução (ord = ordinario $;$ ast = alto sul tasto $;$ asp $=$ alto sul ponticello; $\mathrm{bb}=$ behind the bridge; arthm = artificial harmonic; trem $=$ tremolo; $\mathrm{cr}$ $=$ crushed $; \mathrm{szf}=$ sforzando $;$ tratto = col legno tratto $;$ nonvib = non vibrato $;$ sord $=$ sordina $;$ pizzliv = pizzicato laisser vibrer).

Na Figura 6, apresentamos 03 excertos de “Lana Tai” que estão relacionados com: a) a utilização dos Marcos Sonoros, em uníssono, no começo da obra (Figura 6, a); b) em regiões graves próximo do meio da obra (Figura 6,b) e; c) em regiões mais agudas no fim da obra (Figura 6, c). Na transcrição para a partitura, utilizou-se cada um dos MS de forma a relacionar os instrumentos participantes e as técnicas estendidas (vide Figura 5) com um processo específico de escrita do compositor, alternando e variando as durações, as figurações rítmicas e o tempo de permanência, dado em número de compassos.

a)

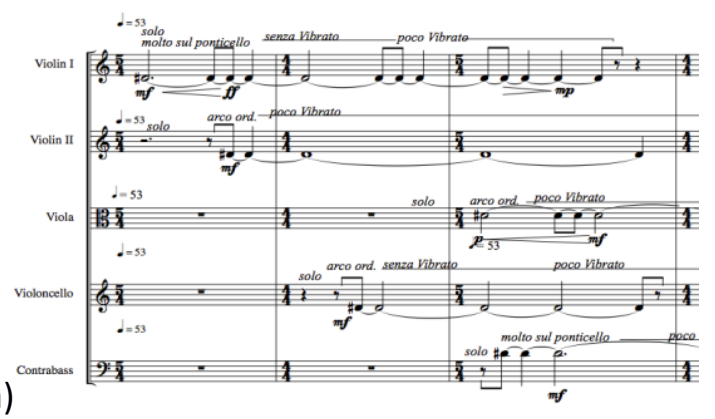

b)
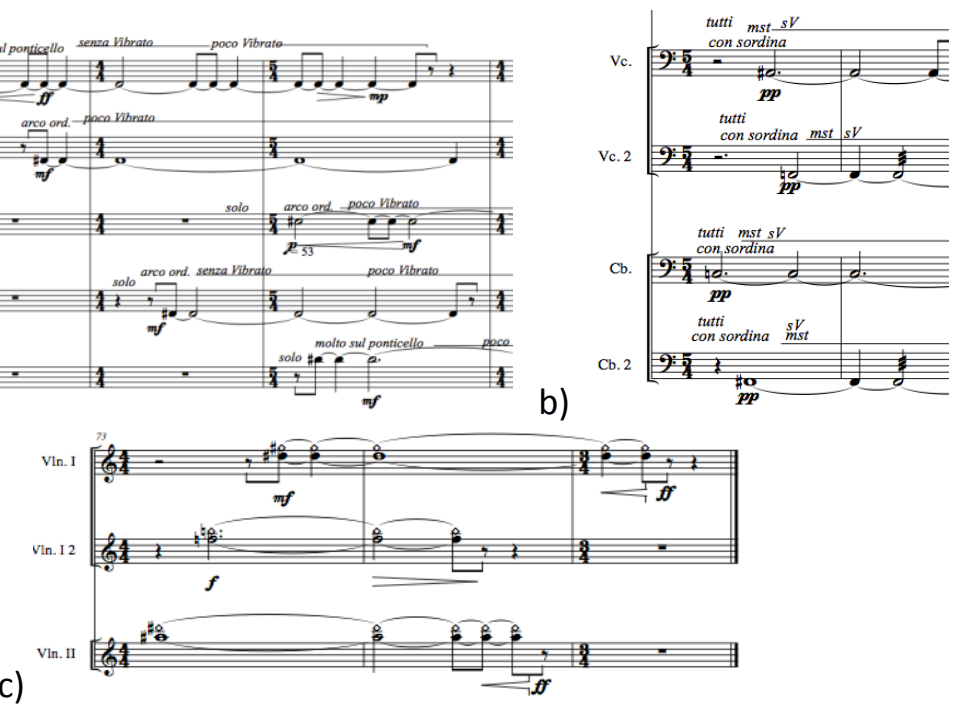

Figura 6: Figura de excertos das três etapas estruturais da sonoridade global de "Lana Tai”.

A obra "Lana Tai" começa em uníssono com apenas um instrumento de cada naipe executando a nota Ré\#4 (Figura 6, a). Iniciou-se a obra com o mínimo de multiplicidade de timbre e, a partir daí, transformou-se a sonoridade da orquestra, de modo a torná-la mais complexa. Para esse desenvolvimento, foram utilizadas diversas técnicas estendidas de execução instrumental e aumentou-se gradativamente o número de alturas diferentes e a 
tessitura de cada naipe. Propiciou-se uma maior distribuição de notas da escala cromática pelos naipes da orquestra. Tal procedimento, como já mencionado anteriormente, foi tratado como uma trajetória e dividiu-se em 3 etapas, nas quais a) inicia-se numa frequência comum a todos os instrumentos, ou seja, o Ré\#4 $\sim 311 \mathrm{~Hz}$; b) desloca-se para uma região grave, até o MS 6 executado por contrabaixos e violoncelos, Fá\#1 46 Hz e, por fim; c) move-se para a região mais aguda, até o MS 15, com os violinos executando Ré\#7 2489 Hz (essas três etapas estão relacionadas com o diagrama da Figura 3).

\section{5 - Discussão}

De modo sucinto, este artigo apresenta uma metodologia para orquestração assistida por computador na qual descrições de alto-nível foram relacionadas à execução instrumental para caracterizar, via descritores de áudio, duas tipologias de sonoridades. A discussão apresentada nos próximos parágrafos tem objetivo de verificar os resultados desse método utilizando o Croma para discutir a interação entre as classes de altura e a complexidade timbrística dos MS. Verificamos, ainda, como os valores do Centroide caracterizam as variações das duas sonoridades em cada MS relacionadas com a estrutura formal da obra.

\section{1 - Polarização e o Croma}

A tabela da Figura 5 está associada aos valores extraídos do Croma para cada uma das 12 classes de alturas dos 15 MS. A partir dessa tabela construímos o gráfico da Figura 7, que descreve mais detalhadamente essas 15 distribuições. As gradações associadas aos quadrados brancos e pretos, indicam os valores da pertinência de cada classe de altura. Os valores foram normalizados no intervalo [0...1]. A variação de cinza está associada respectivamente ao valor mínimo "zero" para quadros pretos e o valor máximo "um” para quadros brancos. Verifica-se nesse gráfico que há uma polarização entre as notas Ré\#, Lá\#, Fá e Si. 


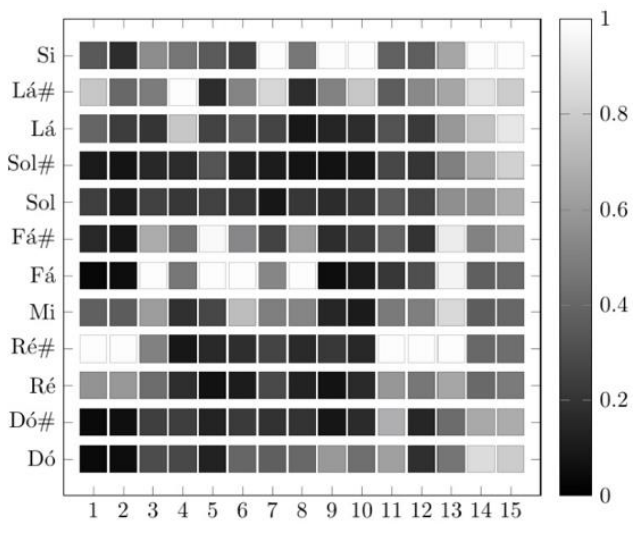

Figura 7: Gráfico de Croma dos 15 MS. As linhas são associadas às classes de altura e as colunas aos MS.

Os dados gerados pelo Croma, na Figura 7, descrevem como foi construído o aumento na complexidade espectral global de "Lana Tai". A partir do MS 3, o valor do Croma associado à nota Fá ou Mi\#, nono componente espectral da fundamental Ré\#, acumula energia até o MS 8. Com a presença, cada vez mais proeminente de reiterações harmônicas da nota Fá, outras classes foram utilizadas pelo compositor, como o aparecimento do Dó no MS 8, no qual os instrumentos tocam Fá em alto sul tasto e pizzicato laisser vibrer e o contrabaixo executa um Dó. A Figura 8, a seguir, representa o excerto executado pelo MS 8.

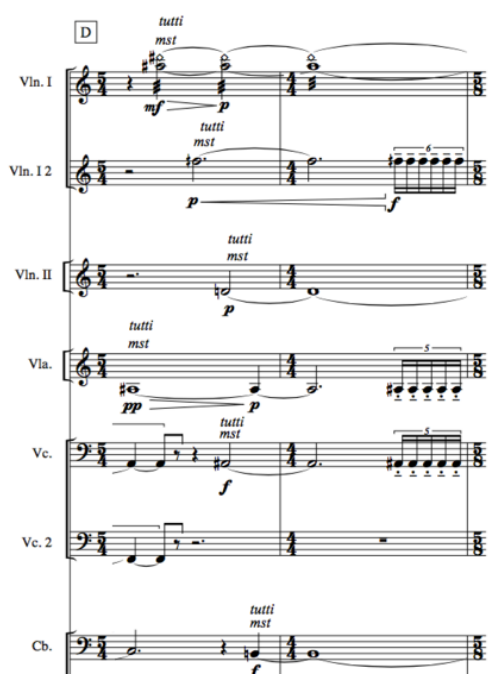

Figura 8: Figura de excerto do MS 8, com apresentação de Dó, no contrabaixo.

A tabela da Figura 7 ilustra que as classes de alturas mais distantes do Ré\#, só obtiveram acúmulos de energia, a partir do MS 13. Ou seja, a medida que a trajetória dirigiu-se para o agudo utilizou-se componentes espectrais mais distantes. Num segundo ponto de análise, 
podemos descrever a média de energia acumulada nas 12 classes de altura do Croma. Com esse procedimento, teremos uma ideia global de como os componentes espectrais estão correlacionados na obra. Para realizar essa análise, calculamos o histograma que integra todos os valores $\mathrm{P}_{\mathrm{i}}$ com $\mathrm{i}=1 \ldots 12$, acumulados nos 15 Marcos Sonoros, utilizando Eq. 6 a seguir:

$$
P_{i}=\frac{1}{15} \sum_{j=1}^{15} D_{i}^{j}(6)
$$

com $i=1 \ldots 12, \mathrm{j}=1 . .15$ e os valores $D_{j}^{i}$ foram retirados da tabela da Figura 8 . Ou seja, uma média da energia acumulada em cada marco sonoro. O resultado dessa formulação é apresentado na Figura 9:

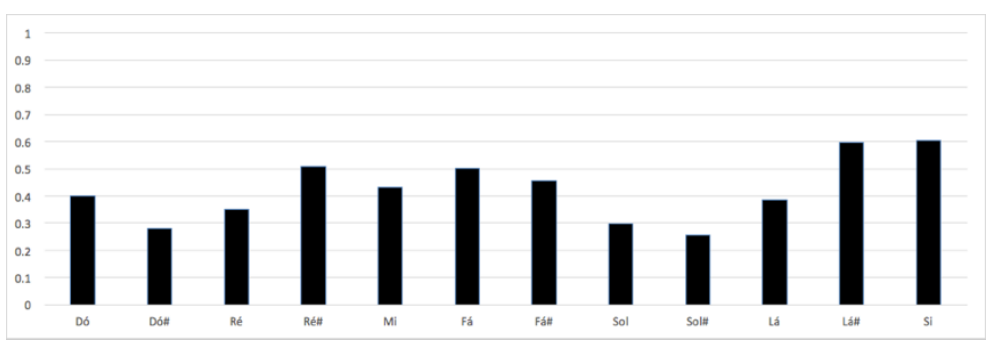

Figura 9: Histograma com a média das magnitudes de cada classe de altura dos MS.

A Figura 9 mostra o acúmulo de energia em classes de alturas das quais relacionam-se diretamente com a nota Ré\#. Além disso, ressaltou-se alguns componentes ímpares da nota fundamental, especialmente o quinto, o nono, o décimo terceiro e o décimo nono componentes espectrais, ou seja, Lá\#, Mi\#, Si e Fá\# respectivamente. O aumento de energia da nota $\mathrm{Si}$, a partir do MS 3, foi ocasionado pela escolha do compositor que incluiu, arbitrariamente, uma classe de altura para manipular o comportamento do Centroide, característica timbrística discutida a seguir. Na Figura 10, apresentamos essas características dos 15 MS em notação musical. Descrevemos a série harmônica do Ré\# e destacamos as notas polarizadas que foram evidenciadas pela análise da distribuição do Croma.

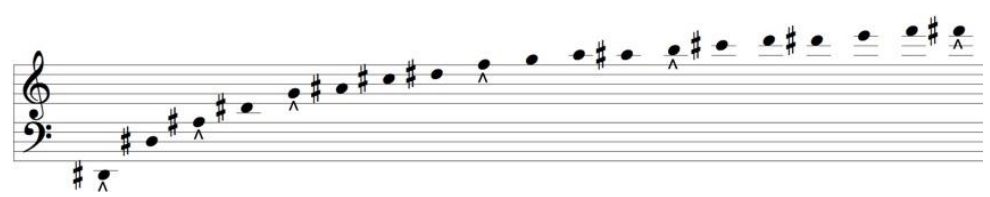

Figura 10: Polarização dos componentes espectrais da nota fundamental Ré\#. As marcações indicam as notas evidenciadas pela média do Croma, em todos os MS. 
Ao utilizar o descritor Croma, no processo composicional, relacionamos as classes de altura com as técnicas estendidas. Criou-se uma dependência direta entre o conteúdo do espectro sonoro com as representações simbólicas utilizadas na transcrição da partitura. Esse processo ampliou, via técnicas instrumentais estendidas, as possibilidades timbrísticas contidas na referência espectral da nota Ré\#, previamente estabelecida. Assim, a paleta sonora da orquestra de cordas foi ampliada com as técnicas de execução contrastantes, como nas regiões graves, agudas e nas diversas possibilidades de inserção de sons não idiomáticos.

\section{2 - Sonoridades e o Centroide}

Do ponto de vista das características timbrísticas extraídas com o Centroide, as três principais etapas estruturais do planejamento composicional de "Lana Tai", foram ressaltadas nos MS 1, MS 6 e MS 15 como descrito no histograma da Figura 11. Vê-se claramente o perfil de variação do Centróide que, entre os MS 01 e 10 tem a tendência de diminuir o seu valor e depois há um aumento gradual entre os MS 11 e 15.

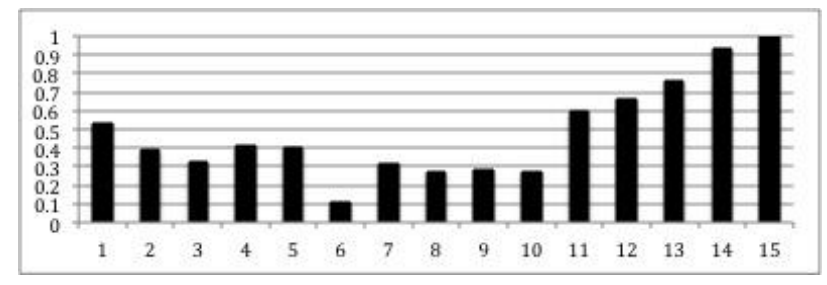

Figura 11: Histograma com os valores de Centroide Espectral de cada MS.

A variação do histograma do Centroide reflete o ideal arquitetado nos Pontos de Referência e construídos nos 15 Marcos Sonoros. A partir desse perfil foi possível descrever a estrutura formal da obra, à qual organiza-se em três grupos distintos, os quais iniciam-se nos MS 01, MS 06 e MS 11. Cada conjunto possui 05 Marcos Sonoros. Há uma ruptura na transição entre os três conjuntos. Pela Figura 11, há uma diferença de aproximadamente $20 \%$ entre o MS 05 e MS 06 e de 30\% entre MS 10 e MS 11. Optou-se por tal construção para evidenciar as três etapas descritas na Subseção 4.2. A tabela da Figura 12 descreve sucintamente a estrutura formal da obra tomando como referência os valores do Centroide. 


\begin{tabular}{|c|c|c|c|c|}
\hline \multicolumn{4}{|c|}{ Estrutura Formal de "Lana Tai - no dia em que nasceu uma aquarela" } \\
\hline Etapa 1: Uníssono & \multirow{2}{*}{ Ruptura } & Etapa 2: Região mais grave & \multirow{2}{*}{ Ruptura } & Etapa 3: Região mais aguda \\
& & & MS 6-MS 10 & \\
\hline
\end{tabular}

Figura 12: Estrutura formal de "Lana Tai", a partir dos conjuntos organizados pelos Pontos de Referência.

A título de exemplo de como a orquestração relacionou-se com o Centroide, destacamos que os instrumentos mais graves, os violoncelos e os contrabaixos, foram executados com sordina, molto sul tasto e com dinâmicas musicais em pianíssimos ou pianississimos, em trechos nos quais há baixos índices de Centroide (vide MS 6). Isso ocorreu para que as implementações técnicas não enfatizassem componentes espectrais de alta frequência. Pois tanto a sordina quanto a técnica de execução em molto sul tasto, inibem a presença desses componentes espectrais de alta frequência.

Finalmente, podemos integrar na nossa discussão a relação entre a polarização relacionada ao Croma e o contraste de sonoridade do Centroide. No início da obra, em uníssono, os primeiros componentes espectrais da classe de altura "Ré\#”' enfatizam o acorde "Ré-SolLá\#. Na tabela da Figura 5, verifica-se que, quando o violino executa a nota Si atrás do cavalete e o contrabaixo executa a nota Si em alto sul tasto, há uma diminuição do valor do Centroide (MS3). No MS 6, ao qual possui o menor índice de Centroide, há ênfase no acorde Fá-Dó-Lá\#. Já no MS 15, no qual explora-se a região mais aguda da obra, não há polarização de uma nota, resultando numa maior dispersão da energia nas classes de alturas musicais. Na Figura 13, há três histogramas da distribuição do Croma que descrevem o resultado sonoro nos MS 1, 6 e 15. À esquerda, o histograma descreve a forte polarização entre Ré\# e Lá\# do MS 1. No centro, há a distribuição do MS 6 na qual obteve, como resultado sonoro, a polarização do acorde Fá-Dó-Lá\#. À direita, o MS 15, no qual utilizou-se o acorde Ré\#Fá-Lá\#, resultou em maior dispersão no acúmulo de energia nas classes de alturas musicais. 


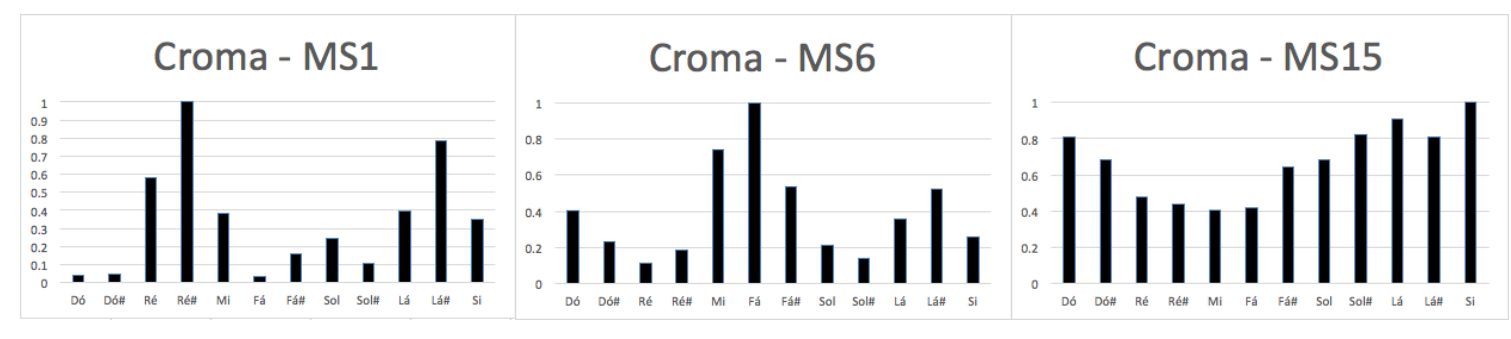

Figura 13: Histogramas do comportamento do Croma em três momentos estruturais de "Lana Tai".

\section{6 - Conclusão}

Apresentamos um estudo sobre a aplicação de descritores de áudio em orquestração assistida por computador visando a composição de uma obra para orquestra de cordas. A motivação para criar-se "Lana Tai-no dia em que nasceu uma aquarela" é o estudo de técnicas MIR como ferramenta para criação musical, abordagem pesquisada no doutorado em desenvolvimento na UNICAMP. A metodologia aqui apresentada versou sobre o planejamento de Pontos de Referência e a construção de Marcos Sonoros que foram ancorados em dois descritores de áudio: Croma e Centroide. Assim, partindo de dois universos: as técnicas de execução instrumental estendidas e os descritores de áudio, desenvolvemos um método para auxiliar o compositor a relacionar descrições de alto nível com modos específicos de execução instrumental estendida. Focou-se a caracterização do timbre sonoro/musical relacionado-o às técnicas de execução instrumental e as suas respectivas orquestrações com o objetivo de obter controles e manipulações mais refinadas.

A partir do método aqui apresentado, pretendemos ampliar o estudo em duas direções: a) criar novos estudos composicionais nos quais possamos explorar outras sonoridades e sempre visaremos à composição de obras para instrumentos executados com técnicas estendidas e b) ampliar a análise dos resultados utilizando também os descritores de áudio para estudar a execução das obras depois de gravadas. O procedimento metodológico apresentado neste artigo, apresenta-se como uma nova estratégia sobre composição e 
orquestração quando explora-se o vasto universo das técnicas estendidas de execução musical.

\section{Referências}

1. ASSAYAG, G.; RUEDA, C.; LAURSON, M.; AGON, C.; DELERUE, O. (1999). Computer-assisted composition at ircam: From patchwork to openmusic. Computer Music Journal, vol. 23, no. 3, p. 59 - 72.

2. BALLET, G.; BORGHESI, R.; HOFFMANN, P.; LÉVY, F. (1999). Studio Online 3.0: An Internet "Killer Application" for Remote Access to IRCAM Sounds and Processing tools. Journées d'Informatique Musicale. France.

3. BARBEDO, J. G. A.; LOPES, A. (2007). Automatic genre classification of musical signals. EURASIP Journal on Applied Signal Processing, v. 2007, n. 1, p.157.

4. BERLIOZ, H. (1844) Treatise on Instrumentation by Hector Berlioz and Richard Strauss, translated by Theodore Front, published by Edwin F. Kalmus, NY, NY 1948.

5. BOGDANOV, D.; WACK, N.; GÓMEZ, E.; et al. (2013). Essentia: An audio analysis library for music information retrieval. Proceedings of ISMIR.

6. BOULEZ, P. (1987). Timbre and composition - timbre and language. Contemporary Music Review. Vol. 2. Translated by R. Robertson. p.161-171

7. BYRD, D; FINGERHUT, M. (2002). The History of ISMIR - A Short Happy Tale. Dlib Magazine, Vol. 8, No. 11.

8. CARPENTIER, G. (2008). Approche computationelle de l'orchestration musicale Optimization multicritère sous contraintes de combinaisons instrumentales dans de grandes banques de sons. Ph.D. Thesis, University Pierre et Marie Curie, Paris.

9. CARTWRIGHT, M.; PARDO, B. (2013). Social-Eq: Crowdsourcing An Equalization Descriptor Map. n. $14^{\circ}$ International Conference - ISMIR. Curitiba - Brazil.

10. DESHPANDE, H.; SINGH, R.; NAM, U. (2001). Classification of music signals in the visual domain. Proceedings of the Conference on Digital Audio Effects. p.1-4.

11. DIELEMAN, S.; SCHRAUWEN, B. (2013). Multiscale Approaches To Music Audio Feature Learning. $14^{\circ}$ International Conference - ISMIR - Curitiba - Brazil.

12. DOWNIE, J. S.; EHMANN, A. F.; BAY, M.; JONES, M. C. (2010). The Music Information Retrieval Evaluation eXchange: Some Observations and Insights. International MIR Systems Evaluation Laboratory. University of Illinois.

13. ESLING, P.; AGON, C. (2013). Multiobjective Time Series Matching for Audio Classification and Retrieval. IEEE Transactions on Audio, Speech, and Language Processing, v. 21, n. 10, p.2057-2072. 
14. ESLING, P.; AGON, C. (2010). Composition of sound mixtures with spectral maquettes. Ann Arbor, MI: MPublishing, University of Michigan Library.

15. ESLING, P.; CARPENTIER, G.; AGON, C. (2010). Dynamic musical orchestration using genetic algorithms and a spectro-temporal description of musical instruments. Applications of Evolutionary Computation. p.371-380. Springer.

16. FUJISHIMA, T. (1999). Realtime Chord Recognition of Musical Sound: a System Using Common Lisp Music. CCRMA, Stanford University. 4 pgs.

17. GOMEZ, E. 2006. Tonal description of music audio signals, Ph.D. dissertation, MTG, Universitat Pompeu Fabra, Barcelona, Spain.

18. HARVEY, J. (1980). The Composer's View: Atonality. The Musical Times, p.699.

19. HUMMEL, T. A. (2005). Simulation of human voice timbre by orchestration of acoustic music instruments. Proceedings of ICMC. p.185.

20. LAMBROU, T.; KUDUMAKIS, P.; SPELLER, R.; SANDLER, M.; LINNEY, A. (1998). Classification of audio signals using statistical features on time and wavelet transform domains. Acoustics, Speech and Signal Processing. Proceedings of the 1998 IEEE International Conference on. v. 6, p.3621-3624.

21. LAURSON, M., KUUSKANKARE, M. (2002). PWGL: A Novel Visual Language based on Common Lisp, CLOS and OpenGL. In Proceedings of International Computer Music Conference, Gothenburg, Sweden. p.142-145.

22. MALT, M.; JOURDAN, E. (2008). Zsa.Descriptors: a library for real-time descriptors analysis. In $5^{\text {th }}$ Sound And Music Computing Conference, Berlin, Allemagne.

23. MALT, M.; JOURDAN, E. (2009). Real-Time Uses of Low Level Sound Descriptors as Event Detection Functions Using the Max/MSP Zsa.Descriptors Library. SBCM, Recife, Brazil.

24. MANZOLLI, J. (1988). Um Modelo Matemático para Timbre Orquestral. Dissertação de mestrado, IMECC, Unicamp.

25. McADAMS,S. \& BATTIER, M. (2005). Creation and perception of a contemporary musical work: The Angel of Death by Roger Reynolds. Ircam, Paris.

26. MONTEIRO, A. (2012). Criação e Performance Musical no Contexto dos Instrumentos Digitais. Dissertação de Mestrado. Núcleo Interdisciplonar de Comunicação Sonora - NICS/UNICAMP. Universidade Estadual Campinas. 159 pgs.

27. PADOVANI, J.; FERRAZ, S. (2011). Proto-História, Evolução e Situação Atual das Técnicas Estendidas na Criação Musical e na Performance. Música Hodie, Vol.11.

28. PAMPALK, E. (2006). Computational models of music similarity and their application in music information retrieval. Tese de Doutorado. Austria: Vienna University of Technology. 
29. PEETERS, G. (2004). A Large Set of Audio Features for Sound Description (Similarity and Classification) in the CUIDADO Project, CUIDADO. Project Report.

30. PEREIRA, E. (2009). Estudos Sobre uma Ferramenta de Classificação Musical. Dissertação de Mestrado-Universidade Estadual Campinas, FEEC. 65 pgs.

31. PIRES, A. S. (2011). Métodos de segmentação musical baseados em descritores sonoros. Dissertação de Mestrado, Universidade de São Paulo.

32. PISTON, W. (1955). Orchestration. W Norton \& Company Incorporated.

33. PUCKeTTE, M. S. (1996). Pure Data. Proceedings, International Computer Music Conference. San Francisco: International Computer Music Association. p.269-272.

34. PSENICKA, D. (2003). Sporch: An algorithm for orchestration based on spectral analyses of recorded sounds. Proceedings of ICMC. p.184.

35. ROSE, F.; HETRICK, J. E. (2009). Enhancing orchestration technique via spectrally based linear algebra methods. Computer Music Journal, v. 33, n. 1, p. 32-41.

36. RIMSKY-KORSAKOV, N. (1922). Principles of Orchestration. ed. by Maximilian Steinberg. Traduzido por Edward Agate; New York: Dover Publications.

37. SCHAFER, M. (1997). A afinação do mundo. Título original em inglês: The Tuning of the World. 1977. Editora UNESP.

38. SHEH A, ELLIS D.P.W. (2003). Chord segmentation and recognition using EMtrained hidden Markov models. International Conference On MIR, Baltimore, USA.

39. SHEPARD, R. N. (1982). Structural representations of musical pitch. In Deutsch, D., editor, The Psychology of Music, First Edition. Swets \& Zeitlinger.

40. THEODORIDIS, S.; KOUTROUMBAS. (2009). Pattern Recognition. Academic press. 967 pgs.

41. THOSHKAHNA, B.; NSABIMANA, F.; RAMAKRISHNAN, K. R. (2011). A Transient Detection Algorithm For Audio Using Iterative Analysis Of Stft. 12th International Society for MIR Conference.

42. WANG, A. (2003). An Industrial Strength Audio Search Algorithm. ISMIR. 
Notas sobre os autores:

Ivan Eiji Simurra é compositor, pesquisador e Dj vinculado ao Núcleo Interdisciplinar de Comunicação Sonora (NICS-UNICAMP). Estudou composição com Silvio Ferraz, Denise Garcia, José Augusto Mannis, Jônatas Manzolli e Eduardo Álvares. Suas obras são tocadas no Brasil, Argentina, Chile, Estados Unidos, Portugal, Irlanda, Israel, Grécia e Rússia. Atualmente, desenvolve sua pesquisa de doutoramento, em Processos Criativos, sob a orientação do Prof. Dr. Jônatas Manzolli e com auxílio da FAPESP.

Jônatas Manzolli é graduado em Matemática Aplicada Computacional (1983) e em Composição e Regência (1987) e mestre em Matemática Aplicada (1988) ambos pela Unicamp e é PhD pela University of Nottingham (1993) no contexto da composição musical. Atualmente é Professor Titular do Instituto de Artes da Unicamp. Compositor e matemático, pesquisa a interação entre Arte e Tecnologia entrelaçando criação musical, computação musical e ciências cognitivas. Sua produção artística relaciona música instrumental, eletroacústica, obras multimídia para dança e instalações sonoras. 